\title{
Transdermal System Dosage Form
}

National Cancer Institute

\section{Source}

National Cancer Institute. Transdermal System Dosage Form. NCI Thesaurus. Code C149999.

Assembly of components intended for transdermal delivery driven by external forces (e.g., electrical current, chemical reaction). 